\title{
Modelo sociocultural del dominio moral en adolescentes mexicanos
}

\author{
Rubén Andrés Miranda-Rodríguez; Mirna García-Méndez
}

Cómo citar este artículo:

Miranda-Rodríguez, R. A., \& García-Méndez, M. (2021). Modelo sociocultural del dominio moral en adolescentes mexicanos. Acta Colombiana de Psicología, 24(2), 69-79. https://www.doi.org/10.14718/ACP.2021.24.2.7

Recibido, mayo 28/2020; Concepto de evaluación, febrero 09/2021; Aceptado, mayo 05/2021

\author{
Rubén Andrés Miranda-Rodríguez ${ }^{1}$ \\ ORCID: https://orcid.org/0000-0003-2734-6856 \\ Universidad Nacional Autónoma de México, Ciudad de México, México. \\ Mirna García-Méndez \\ ORCID: https://orcid.org/0000-0002-2334-0740 \\ Universidad Nacional Autónoma de México, Ciudad de México, México.
}

\begin{abstract}
Resumen
Para favorecer el desarrollo de la adolescencia es fundamental fomentar un dominio moral que tenga en cuenta las características de la cultura y la dinámica familiar, pues ambas permiten comprender los principios que conforman el juicio moral. Teniendo esto en cuenta, la presente investigación tuvo como objetivo determinar si el colectivismo, las premisas históricosociales-culturales y el funcionamiento familiar predicen el dominio moral en adolescentes mexicanos. Para ello, se contó con una muestra de 459 adolescentes que participaron de manera voluntaria en la aplicación de la Escala de Dominio Moral para Adolescentes, una adaptación de la Escala de Premisas Histórico-Socioculturales, y la Escala de Individualismo y Colectivismo, en secundarias o preparatorias públicas de Ciudad de México y municipios del Estado de México. El análisis de los resultados se realizó con ecuaciones estructurales, tras lo cual se obtuvo un modelo en el que el colectivismo, las normas tradicionales de la cultura mexicana y el funcionamiento familiar predicen el dominio de una moral prescriptiva y restrictiva en adolescentes mexicanos $\left(\chi^{2}=0.496, \mathrm{gl}=1, p=.481, \chi^{2} / \mathrm{gl}=0.496, \mathrm{AGFI}=.992, \mathrm{SRMR}=.006, \mathrm{RMSEA}<.001\right)$. En conclusión, la evidencia obtenida demuestra que una parte importante de las variaciones del dominio moral en adolescentes se debe a factores culturales y de socialización en el entorno familiar.

Palabras clave: adolescentes, dominio moral, cultura, funcionamiento familiar, desarrollo.
\end{abstract}

\section{Sociocultural model of moral domain in Mexican adolescents}

\begin{abstract}
In order to favor the development of adolescence it is essential to foster a moral domain that takes into account the characteristics of culture and family dynamics, since both allow to understand the principles that shape moral judgement. This research aimed to determine whether collectivism, historical-socio-cultural premises and family functioning predict moral domain in Mexican adolescents. For this purpose, a sample of 459 adolescents participated voluntarily in the application of the Moral Domain Scale for Adolescents, an adaptation of the Historical-Social-Cultural Premises Scale, and the Individualism and Collectivism Scale, in public secondary schools or high schools in Mexico City and municipalities of the State of Mexico. Data were analyzed using structural equations modeling. A model was obtained in which collectivism, the traditional norms of Mexican culture and family functioning predict the domain of a prescriptive and restrictive morality in Mexican adolescents. In conclusion, the evidence found shows that an important part of the variations in the moral domain in adolescents is due to cultural and socialization factors in the family environment. Keywords: adolescents, moral domain, culture, family functioning, development.
\end{abstract}

\footnotetext{
1 Av. Guelatao No. 66 (Eje 7 Oriente), colonia Ejército de Oriente, Ciudad de México. C.P.: 09230. Tel.: 5518190776. r.andresmiranda.r@gmail.com

Nota del autor: se agradece al Consejo Nacional de Ciencia y Tecnología (CONACYT) de México por la beca otorgada al primer autor, con la que se financió la presente investigación.
} 


\section{Introducción}

Un indicador psicológico del bienestar y la adaptación social en adolescentes es la capacidad de distinguir lo moralmente correcto e incorrecto, ya que esto les permite aprender a regular sus comportamientos a partir de criterios que eviten daños potenciales a sí mismos o a otros seres vivos. Por esta razón, un elemento fundamental durante la adolescencia es la formación de un criterio moral consistente con la preocupación social, a saber: el aprendizaje de principios morales que los involucren con las necesidades de su comunidad (Martin, 2006).

Para promover esta formación, resulta necesario identificar y conocer la orientación cultural de los adolescentes por medio de las creencias, costumbres y tradiciones que le dan significado a los principios morales que se desean promover (Barrios et al., 2012), y para ello se debe primero clarificar lo que significa cada concepto, tal como veremos a continuación.

Inicialmente, un principio moral se entiende como una afirmación normativa sobre las acciones buenas y malas, con la característica de que se desarrolla a través de la experiencia (Railton, 1986). En un contexto más amplio, la educación moral hace referencia a la promoción de principios morales para beneficio de una sociedad, y esta se desarrolla teniendo en cuenta tanto el entorno sociocultural como los sentimientos y pensamientos de las personas a las que se desea educar (Buxarrais-Estrada \& Martínez-Martín, 2009), por lo que es importante que se tome en cuenta un enfoque sociocultural como alternativo a un enfoque universalista (Haste, 2013). Y, por otra parte, al conjunto de principios morales que rigen y regulan el comportamiento humano se le denomina dominio moral, que refiere a aquel que incorpora las afirmaciones dadas por hecho en la realidad sociocultural de las personas y forma las bases de sus criterios sobre lo bueno y lo malo (Turiel, 1983).

De hecho, el principal referente del estudio del dominio moral se encuentra en la teoría de Kohlberg (1987), en la cual se describe una clasificación en seis niveles que definen diferentes maneras de razonar conforme a los siguientes principios morales, a saber: (a) la moral heterónoma, en la que se razona conforme a consecuencias físicas y el juicio de otros, normalmente de alguna autoridad; (b) la moral individualista instrumental, en la que se razona conforme a las necesidades y deseos únicamente personales; (c) la moral normativa interpersonal, en la que se razona conforme a las expectativas del entorno social; (d) la moral de sistema social, en la cual se razona conforme al cumplimiento de normas, reglas o leyes establecidas; (e) la moral del contrato social, donde se razona conforme a los derechos humanos y la búsqueda de acuerdos que beneficien a todos; y (f) la moral de principios éticos universales, en la cual se razona conforme a la propia conciencia sobre ciertos principios universales, como lo son el bienestar y la justicia.

Ahora bien, sobre la base de un dominio moral compuesto por los principios de la teoría de Kohlberg se han desarrollado diferentes instrumentos de evaluación psicológica, y uno de los más utilizados es el Defining Issues Test (DIT), que mide cómo las personas se acercan o alejan del nivel más alto de desarrollo moral según la manera de razonar ante dilemas morales (Rest, 1979). No obstante, los estudios de Barba (2001, 2002), en los que se adaptaron los dilemas del DIT a la cultura mexicana, muestran que los mexicanos obtienen puntajes más bajos de desarrollo moral en esta prueba en comparación con personas de Estados Unidos, lo que sugiere dos posibles razones: que los estadounidenses tienen un mejor nivel de razonamiento moral que los mexicanos, o que existen aspectos socioculturales que cuestionan si es posible hacer comparaciones pertinentes entre muestras de ambos países.

En términos de la relación entre el dominio moral y la sociocultura, Triandis (1994) afirma que los principios 3 y 4 de la teoría de Kohlberg corresponden más con principios morales de culturas colectivistas, y que los principios 5 y 6 tienen un carácter más individualista. Otros autores, como Shweder et al. (1987), por ejemplo, afirman que los principios 5 y 6 de la teoría de Kohlberg obedecen a principios morales con una lógica liberal de occidente, y que estos no atienden realmente a otras culturas con normas, creencias y valores más enfocados hacia la colectividad o la espiritualidad.

En este sentido, Shweder et al. (1987) sugieren que el dominio moral varía culturalmente en tres grandes tendencias: (a) en la autonomía, en función de principios morales que valoran la individualidad y la independencia; (b) en la comunidad, en función de principios morales que valoran la pertenencia a grupos y la interdependencia; y (c) en la divinidad, en función de principios morales que valoran la espiritualidad o la religiosidad. Estas posturas alternativas sugieren que el dominio moral desde un enfoque completamente universal no puede aplicarse a todas las culturas, ya que existen diferencias respecto a los ideales morales que imperan en cada entorno social. Así, si la teoría de Kohlberg no considera las diferencias culturales, un dominio moral conformado por principios de autoconsciencia, justicia y bienestar es insuficiente.

Al respecto, Haidt y Joseph (2007) plantean que para establecer un dominio moral aplicable a todas las personas y que atienda a la variabilidad cultural se deben reconocer 
primero sus bases bioevolutivas, es decir, las necesidades que deben satisfacerse para sobrevivir a través de un conjunto de comportamientos sociales adaptativos al ambiente; a estas bases se les denomina fundamentos morales. Con base en estos estudios, Graham et al. (2013) sugieren cinco fundamentos morales que conforman el dominio moral: (a) el de cuidado/daño, entendido como la necesidad de protección y consideración por los vulnerables; (b) el de justicia/injusticia, donde se busca establecer relaciones de reciprocidad y cooperación; (c) el de lealtad/traición, que promueve la conformación y cohesión de grupos; (d) el de autoridad/subversión, que se refiere a la importancia de las jerarquías para la organización social; y (e) el de purezal degradación, en el que se conserva o se rechaza algo según los sentimientos de disgusto o desagrado que generen.

Ahora bien, los fundamentos morales tienen la cualidad de ser universales a nivel teórico por su carácter biológico y evolutivo, pero en lo empírico se debe considerar cómo pueden variar de acuerdo con el entorno sociocultural. Si se trata de un conjunto de bases previas a la interacción de las personas con su medio ambiente, es plausible que se modifiquen de acuerdo con las características del ecosistema en donde se desarrollen (Haste, 2013).

De hecho, esto último se tomó en consideración en un estudio realizado con adolescentes de la Ciudad de México y zonas conurbadas del Estado de México, donde se elaboró una definición de dominio moral en adolescentes mexicanos como un proceso culturalmente variable, en el cual se manifiestan principios morales que dirigen las relaciones sociales hacia la preservación del amor, el respeto mutuo, la lealtad a la familia y la equidad, así como el rechazo a las ofensas, como faltas de respeto a los valores y a las acciones perjudiciales e injustas (Miranda-Rodríguez \& García-Méndez, 2019). Según estos autores, la clasificación de dominio moral en adolescentes mexicanos consiste en cinco principios morales: (a) amor/respeto, un conjunto de juicios que favorecen las muestras de afecto, la preocupación por el bienestar del otro y la demostración de que las personas son valiosas; (b) lealtad/familia, un conjunto de juicios que fortalecen la estabilidad de los preceptos familiares y el valor de la familia como guía moral; (c) ofensa, un conjunto de juicios que reprueban la deshonestidad y la pérdida de valores a través de actos como la mentira y la venganza; (d) equidad, un conjunto de juicios en favor del dar-recibir en las relaciones, la justicia y el trato igualitario para todas las personas, donde se buscan los mismos derechos y condiciones para todos; y (e) perjuicio, un conjunto de juicios que reprueban la voluntad de agredir, provocar dolor y aprovecharse de las condiciones de otros. Estos principios toman en cuenta una perspectiva sociocultural (Miranda-Rodríguez \& García-Méndez, 2019).
Al considerar una perspectiva sociocultural del dominio moral, es fundamental identificar a qué factores locales y específicos se deben las variaciones en los principios morales de una cultura en particular, con la finalidad de estructurar una educación moral sensible a cada sociedad (Berniūnas et al., 2016; Buxarrais-Estrada \& Martínez-Martín, 2009). En este sentido, las Premisas Histórico-Socio-Culturales (PHSC) planteadas por Díaz-Guerrero $(1994,2003)$ como predictoras del dominio moral en adolescentes mexicanos pueden servir para cumplir este propósito, debido a que marcan la orientación moral de las conductas (Díaz-Loving, 2011).

En particular, las PHSC se definen como un conjunto de afirmaciones simples o complejas que marcan las formas de convivencia de los mexicanos (Díaz-Guerrero, 1972, 1994, 2003), que se distribuyen en diferentes tipos de afirmaciones, entre los que se encuentran: (a) la obediencia afiliativa, que refiere a anteponer los intereses propios por los de la familia; (b) el marianismo, una posición tradicional de la mujer en la cultura que involucra el protegerla y cuidarla, y en la que se le otorga como principal función regir el hogar; (c) el honor familiar, la importancia que se le otorga a las relaciones extramaritales como una forma de estigma familiar; (d) el temor a la autoridad, es decir, el grado de aprensión de los hijos hacia los padres; (e) la autoafirmación, la disposición para buscar la autonomía y el autorreconocimiento, como personas independientes de la familia; (f) el statu quo familiar, la tendencia a mantener sin cambios la estructura tradicional de relaciones entre los miembros de la familia; y (g) el consentimiento, el grado de aceptación de la superioridad del hombre sobre la mujer, a quien se coloca en una posición de abnegación y sufrimiento (García-Méndez, 2007). En investigaciones más recientes, estas premisas son clasificadas en dos grandes componentes: (a) normas referidas a la delimitación de lo que se debe hacer; y (b) creencias que son la delimitación de cómo se entienden las relaciones interpersonales y los roles sociales (Díaz-Loving et al., 2011).

Desde esta perspectiva, se considera que las normas y creencias tradicionales de los mexicanos participan en la conformación de sus principios morales debido a aspectos de la cultura local; sin embargo, también se han considerado elementos transculturales en la conformación de la noción del bien y del mal en las personas, tal como se plantea en algunas de las críticas a la teoría de Kohlberg (Shweder et al., 1987). Uno de dichos elementos, considerado como un patrón transcultural que participa en la variación de los principios morales, es el síndrome cultural del individualismocolectivismo (Yilmaz et al., 2016).

Los síndromes culturales se entienden como patrones de comportamiento social que permiten hacer comparaciones entre culturas (Hofstede, 2001), y, en particular, el 
individualismo-colectivismo es un síndrome cultural que involucra la búsqueda de la autonomía e independencia o los deseos de permanecer en grupos de manera interdependiente (García-Campos et al., 2016; Triandis, 1994). Al respecto, se ha demostrado que hay principios morales relacionados tanto con el individualismo - como el cuidado y la justicia - como con en el colectivismo - aspectos de lealtad o autoridad- (Yilmaz et al., 2016), aunque cabe mencionar que los principios morales en adolescentes mexicanos se identifican como colectivistas (MirandaRodríguez \& García-Méndez, 2019), y que el colectivismo es un predictor positivo de aspectos sociales del dominio moral (Sullivan et al., 2012).

De este modo, se estima conveniente identificar la participación del colectivismo como predictor de los principios morales de adolescentes mexicanos en función de que la cultura mexicana es predominantemente colectivista, con la familia como grupo social de referencia (Díaz-Guerrero, 1994). Es por esto que los patrones de socialización y su funcionamiento en el entorno familiar también pueden estar presentes en la variación de principios morales, lo que se suma a que se ha demostrado que un funcionamiento familiar con ambiente positivo tiene efectos positivos en el desarrollo moral de los adolescentes (White \& Matawie, 2004).

Al respecto, el funcionamiento familiar se define como un conjunto de patrones de socialización que se dan entre los integrantes de una familia a lo largo de su ciclo de vida (García-Méndez et al., 2006; Taibo et al., 2018) y que, según Jaen (2017), desde la percepción de los adolescentes se clasifica en cuatro componentes: (a) la cohesión y comunicación, es decir, el intercambio de información entre los miembros familiares, cuya importancia estriba en el conocimiento y claridad del contenido de los mensajes verbales; (b) el esparcimiento y armonía, entendido como la capacidad de los miembros de la familia de relacionarse positivamente fuera del sistema familiar; (c) las coaliciones, que se refieren a la unión de dos o más personas para dañar a alguien más dentro de la familia; y (d) la ausencia de reglas y límites, entendida como los patrones que adopta una familia, donde las reglas y las jerarquías no son claras para los miembros del grupo, porque se trasgreden sin importar si se es hijo/a, padre o madre. Estos patrones de socialización se forjan según los roles establecidos y la influencia de los ambientes sociales en los que se desenvuelven (García-Méndez et al., 2006). En este contexto, para identificar aspectos de socialización que complementen los procesos culturales que participan en la variación del dominio moral, el funcionamiento familiar puede aportar elementos que se puedan considerar como un predictor de esta variable.
Definido lo anterior, el objetivo general de la presente investigación consistió en determinar si las PHSC, el colectivismo y el funcionamiento familiar predicen el dominio moral en adolescentes mexicanos. Así, partiendo de la literatura revisada (Díaz-Loving, 2011; Sullivan et al., 2012; White \& Matawie, 2004; Yilmaz et al., 2016), se sugirieron como hipótesis que: las PHSC, el colectivismo y los componentes positivos del ambiente familiar predicen de manera estadísticamente significativa y positiva al dominio moral en adolescentes mexicanos (H1); mientras que los componentes negativos del ambiente familiar lo hacen de forma estadísticamente significativa y negativa (H2). Finalmente, otro objetivo fue identificar si dichas variables conforman un modelo estructural al compartir cualidades de normas, creencias, costumbres, valores y tradiciones que se transmiten a través de la socialización (Díaz-Guerrero, 2003).

\section{Método}

\section{Tipo de estudio}

Al sugerir un modelamiento estructural, se propuso un estudio con un alcance correlacional multivariado (Ruiz et al., 2010).

\section{Participantes}

Se realizó un muestreo no probabilístico intencional en el que se seleccionaron 459 adolescentes $(58 \%$ mujeres y $42 \%$ hombres), con los criterios de inclusión de estar inscritos en secundarias o preparatorias públicas y laicas, estar en un rango de edad de 12 a 17 años $(M=14.64, D E=1.42)$, y ser residentes de la Ciudad de México o municipios del Estado de México. Los criterios de exclusión fueron tener reportes de comportamiento antisocial o delictivo por parte de las autoridades de su escuela.

\section{Instrumentos}

Los siguientes instrumentos fueron elegidos por su pertinencia para medir las variables propuestas en el modelo y por las propiedades psicométricas que se muestran en sus respectivos estudios originales. A continuación se presenta cada uno de ellos con los índices de confiabilidad globales y por factor reportados en sus estudios de validación.

\section{Escala de Dominio Moral para Adolescentes}

Esta escala, desarrollada por Miranda-Rodríguez y García-Méndez (2019), está compuesta por 20 reactivos distribuidos en cinco factores que explican el $43.07 \%$ de 
la varianza, y cuenta con un alfa de Cronbach global de .84. Los cinco factores que la componen son: amor/respeto (5 reactivos, $\alpha=.75$ ), lealtad/familia (3 reactivos, $\alpha=.70$ ), ofensa ( 3 reactivos, $\alpha=.62$ ), equidad ( 4 reactivos, $\alpha=.63$ ) y perjuicio (5 reactivos, $\alpha=.71)$.

\section{Adaptación de la Escala de Premisas Histórico-Socioculturales}

Una adaptación de la escala realizada por Díaz-Guerrero (2003) y García-Méndez (2007), compuesta por 33 reactivos agrupados en siete factores que explican el $57 \%$ de la varianza, con un alfa de Cronbach global de .82. Los siete factores que componen la escala son: obediencia afiliativa ( 6 reactivos, $\alpha=.83$ ), consentimiento ( 6 reactivos, $\alpha=.81$ ), autoafirmación (4 reactivos, $\alpha=.85$ ), status quo (6 reactivos, $\alpha=.72$ ), temor a la autoridad (4 reactivos, $\alpha=.84$ ), marianismo (4 reactivos, $\alpha=.62$ ), y honor familiar (3 reactivos, $\alpha=.58$ ).

\section{Escala de Individualismo y Colectivismo}

Esta escala, desarrollada por Soler-Anguiano y DíazLoving (2017), está conformada por 31 reactivos que integran cuatro factores que explican el $43.74 \%$ de la varianza, y cuenta con un alfa de Cronbach global de .84. Los cuatro factores que la componen son: colectivismo horizontal (13 reactivos, $\alpha=.84$ ), individualismo horizontal ( 7 reactivos, $\alpha=.77$ ), individualismo vertical (6 reactivos, $\alpha=.76$ ) y colectivismo vertical (5 reactivos, $\alpha=.61$ ).

Escala de Funcionamiento Familiar para Adolescentes Elaborada por Jaen (2017), está compuesta por 25 reactivos que se agrupan en cuatro factores que explican el $54.21 \%$ de la varianza total, y cuenta con un alfa de Cronbach global de .87. Los cuatro factores que componen la escala son: cohesión y comunicación (13 reactivos, $\alpha=.89$ ), esparcimiento y armonía (6 reactivos, $\alpha=.82$ ), coaliciones ( 3 reactivos, $\alpha=.72$ ), y ausencia de reglas y límites (3 reactivos, $\alpha=.64$ ).

\section{Procedimiento}

Para obtener los datos se acudió a diferentes secundarias y preparatorias de la Ciudad de México y municipios del Estado de México, donde se solicitaron los permisos para la aplicación de los instrumentos a los directores de las escuelas, quienes, tras la explicación de los objetivos de la investigación, otorgaron su consentimiento informado después de revisar los instrumentos.

Las aplicaciones se realizaron de manera grupal en los salones de clase, con la supervisión de los investigadores y una autoridad respectiva encargada de cada grupo, como profesores o tutores. Cada adolescente contó con un asentimiento de participación en el que tenía la libertad de decidir si deseaba o no contestar los instrumentos. Asimismo, se garantizó su anonimato y confidencialidad.

Después de cada aplicación, se agradeció a los adolescentes y autoridades su colaboración y se proporcionaron datos de contacto para cualquier aclaración. La investigación fue revisada y aprobada por el comité de bioética de la Facultad de Estudios Superiores Zaragoza de la Universidad Nacional Autónoma de México.

\section{Análisis de datos}

Debido a que los instrumentos ya cuentan con estructuras factoriales definidas, se emplearon cuatro análisis factoriales confirmatorios con el fin de simplificar los resultados en un solo modelo predictivo. De la escala de premisas histórico-socio-culturales se analizó una estructura de dos factores con el propósito de adaptar las características de la escala a las sugerencias de clasificación de las PHSC como normas y creencias (Díaz-Loving et al., 2011). De las escalas de dominio moral, individualismo-colectivismo y funcionamiento familiar se realizaron análisis factoriales de segundo orden.

Una vez obtenidos los factores de segundo orden de las escalas, se realizaron correlaciones producto momento de Pearson y análisis de regresión lineal múltiple para identificar las variables que se relacionan y predicen al dominio moral en adolescentes, e incluirlas en el modelo estructural. También, se llevaron a cabo las pruebas para corroborar supuestos de normalidad, homocedasticidad, colinealidad e independencia de las variables predictoras.

Para establecer el modelo estructural se realizó un modelamiento por ecuaciones estructurales, debido a que este análisis permite integrar los resultados con base en la hipótesis de que las variables predictoras y predichas participan en un modelo conjunto que contempla los errores de medición que se restringen en los análisis de regresión (Ruiz et al., 2010).

Finalmente, para los análisis factoriales confirmatorios y la comprobación del modelo estructural se consideraron índices basados en reglas sobre el modelamiento por ecuaciones estructurales (Hu \& Bentler, 1999; Ruiz et al., 2010), y se utilizaron los criterios de Chi cuadrada sobre grados de libertad $\left(\chi^{2} / \mathrm{gl}\right)$ con valores esperados menores a 3, el Adjusted Goodness of Fit Index (AGFI) con valores esperados mayores a .95 y mayores a .90 como aceptables, el Standardized Root of Mean Squared Residual (SRMR) con valores esperados menores a .08, y el Root Mean Square 
Error of Approximation (RMSEA) con valores esperados menores a .06 y menores a .08 como aceptables. Todos los datos se analizaron con los programas SPSS y AMOS versión 24 .

\section{Resultados}

Inicialmente, respecto al análisis factorial confirmatorio de la escala de premisas histórico-socio-culturales, se obtuvo una versión corta de 15 reactivos distribuidos en dos factores, denominados normas (con 9 reactivos sobre la obediencia afiliativa, el temor a la autoridad y el honor familiar, $\alpha=.85$ ) y creencias (con 6 reactivos sobre el status quo, la autoafirmación y el marianismo, $\alpha=.80$ ), que explican el $42.6 \%$ de la varianza y presentan un coeficiente global $\alpha=.80$. Los índices de ajuste obtenidos fueron aceptables $\left(\chi^{2}=189.117, \mathrm{gl}=87, p<.001, \chi^{2} / \mathrm{gl}=2.174\right.$, AGFI $=.927$, SRMR $=.048$, RMSEA $=.051$ ).

Por otra parte, con la escala de dominio moral se obtuvieron dos factores supraordenados conceptuados con base en la teoría (McKinney, 1971) que explican el $51.30 \%$ de la varianza: moral prescriptiva (amor/respeto, lealtad/ familia y equidad), definida como el conjunto de principios morales que delimitan lo que se debe hacer - como amar, respetar, ser leal y equitativo-; y moral restrictiva (ofensa y perjuicio), definida como el conjunto de principios morales que delimitan lo que no se debe hacer - no ofender a otros ni provocarles daños o problemas-. Los índices de ajuste obtenidos fueron aceptables $\left(\chi^{2}=3.277, \mathrm{gl}=2, p=.194\right.$, $\chi^{2} / \mathrm{gl}=1.639, \mathrm{AGFI}=.978$, SRMR $=.021$, RMSEA $\left.=.038\right)$.

De igual forma, para individualismo-colectivismo se obtuvieron dos factores: colectivismo (horizontal y vertical) e individualismo (horizontal y vertical), que explican el $73.12 \%$ de la varianza, con índices de ajuste aceptables $\left(\chi^{2}=4.872, \mathrm{gl}=2, p=.08, \chi^{2} / \mathrm{gl}=2.436\right.$, AGFI $=.973$, SRMR $=.030$, RMSEA $=.056) ; \mathrm{y}$ para el funcionamiento familiar también se obtuvieron dos factores: ambiente familiar positivo (cohesión y comunicación y esparcimiento y armonía) y ambiente familiar negativo (coaliciones y ausencia de reglas y límites), que explican el $66.52 \%$ de la varianza, con índices de ajuste también aceptables $\left(\chi^{2}=2.671, \mathrm{gl}=2, p=.263, \chi^{2} / \mathrm{gl}=1.336, \mathrm{AGFI}=.985\right.$, SRMR $=.032$, RMSEA $=.027$ ).

Una vez obtenidos los factores de segundo orden de las escalas, se procedió con las correlaciones y los análisis de regresión lineal múltiple. Como se muestra en la Tabla 1, se encontraron correlaciones estadísticamente significativas y positivas de la moral prescriptiva con las normas, el colectivismo, el individualismo y el ambiente familiar positivo, así como una correlación estadísticamente significativa y negativa con el ambiente familiar negativo; mientras que para la moral restrictiva se encontraron correlaciones estadísticamente significativas y negativas con las normas y el ambiente familiar negativo, así como una correlación estadísticamente significativa y positiva con el colectivismo.

Tomando como base las correlaciones obtenidas, se procedió a realizar dos análisis de regresión lineal múltiple: uno para predecir la moral prescriptiva y otro para predecir la moral restrictiva. Para ello, se efectuaron ambos modelos de regresión y posteriormente se verificaron los análisis en función de los supuestos de normalidad, homocedasticidad, colinealidad e independencia de los errores (Berry \& Feldman, 1985; Cohen et al., 2013; Miles \& Shevlin, 2001). Específicamente, para controlar los supuestos de normalidad y homocedasticidad se analizaron los residuales estandarizados de los datos después de efectuar los modelos de regresión, y se eliminaron cuatro datos considerados atípicos (outliers) $(Z<-4)$, por lo que el tamaño de la muestra quedó de $N=455$.

Después de esto, se realizaron los análisis de regresión lineal múltiple correspondientes. En el primero se identificó al colectivismo como el principal predictor de las variaciones en la moral prescriptiva de acuerdo con el coeficiente beta estandarizado $(\beta)$, seguido de las normas y el ambiente familiar positivo, los tres como predictores estadísticamente significativos y positivos; y el ambiente familiar negativo surgió como predictor estadísticamente significativo y negativo (véase Tabla 2). Esto confirma las hipótesis de que las PHSC, el colectivismo y el ambiente familiar positivo son predictores positivos, así como la hipótesis del ambiente familiar negativo como predictor negativo del dominio moral, precisando que cuando se

Tabla 1.

Correlaciones de los factores de dominio moral con los factores de premisas histórico-socio-culturales, individualismocolectivismo y funcionamiento familiar

\begin{tabular}{ccccccc}
\hline & Normas & Creencias & Colectivismo & Individualismo & $\begin{array}{c}\text { Ambiente fa- } \\
\text { miliar positivo }\end{array}$ & $\begin{array}{c}\text { Ambiente familiar } \\
\text { negativo }\end{array}$ \\
\hline Moral prescriptiva & $.278^{* *}$ & -.001 & $.352^{* *}$ & $.114^{*}$ & $.236^{* *}$ & $-.172^{* *}$ \\
Moral restrictiva & $-.100^{*}$ & -.016 & $.197^{* *}$ & -.041 & .085 & $-.163^{* *}$ \\
\hline
\end{tabular}

Nota. $* * p<.01 ; * p<.05$. 
promueven el colectivismo, las normas tradicionales y un ambiente familiar favorable, probablemente los adolescentes comprendan la importancia de prescribir conductas que impliquen amor, respeto, lealtad a la familia y equidad.

Las pruebas para corroborar el supuesto de colinealidad indicaron que no existe multicolinealidad de las variables predictoras con respecto al modelo de regresión (colectivismo, tolerancia $=.822, \mathrm{VIF}=1.217$; normas, tolerancia $=.884$, $\mathrm{VIF}=1.131 ;$ ambiente familiar negativo, tolerancia $=.974$, $\mathrm{VIF}=1.026$; ambiente familiar positivo, tolerancia $=.857$, $\mathrm{VIF}=1.166$ ); $\mathrm{y}$ las pruebas de independencia de los errores muestran que los residuales de las variables predictoras son independientes (Durbin-Watson value $=1.879$ ).

Posteriormente, en el segundo análisis se realizó el mismo procedimiento y se identificó al colectivismo, nuevamente, como el principal predictor de la moral restrictiva de acuerdo con el coeficiente beta estandarizado $(\beta)$, además de mostrarse como predictor estadísticamente significativo y positivo, seguido del ambiente familiar negativo y las normas, ambos como predictores estadísticamente significativos y negativos (véase Tabla 3). Esto también confirma las hipótesis, pero con la excepción de que las normas participan como un predictor negativo, precisando que cuando se promueve el colectivismo, se regula la comunicación de normas tradicionales y se evita un ambiente familiar negativo, probablemente los adolescentes comprendan la importancia de restringir conductas que impliquen ofender o perjudicar a otras personas.

Además, las pruebas para corroborar el supuesto de colinealidad indicaron que no existe multicolinealidad de las variables predictoras con respecto al modelo de regresión (colectivismo, tolerancia $=.899, \mathrm{VIF}=1.113$; ambiente familiar negativo, tolerancia $=.983, \mathrm{VIF}=1.017$; normas, tolerancia $=.898, \mathrm{VIF}=1.113$ ); $\mathrm{y}$ las pruebas de independencia de los errores muestran que los residuales de las variables predictoras tienen autocorrelación positiva (Durbin-Watson value $=0.002$ ), por lo que no muestran independencia. Sin embargo, se estima que el error de medición se puede deber a una relación teórica entre las variables predictoras con respecto a la variable predicha (Berry \& Feldman, 1985): para los adolescentes, el colectivismo, el ambiente familiar negativo y las normas culturales comparten una lógica particular en relación con una moral restrictiva, que puede deberse a la consideración de la familia como una autoridad moral que marca los límites sociales y culturales de sus conductas (White \& Matawie, 2004).

Más adelante, con la finalidad de examinar los resultados en un solo modelo predictivo, se realizó un modelamiento por ecuaciones estructurales que integra las variables predictoras de la moral prescriptiva y la moral restrictiva. Con base en las regresiones, se propuso un modelo con el colectivismo, las normas, el ambiente familiar negativo y el ambiente familiar positivo como variables predictoras de la moral prescriptiva, y las mismas variables, con excepción del ambiente familiar positivo, como predictoras de la moral restrictiva. Con esto, el modelamiento por ecuaciones estructurales mostró índices de ajuste adecuados con una línea de covariación entre la moral prescriptiva y la moral restrictiva, por lo que se confirma la estimación de que, en conjunto, pueden conformar un modelo estructural

Tabla 2.

Resumen del análisis de regresión lineal múltiple para las variables predictoras de la moral prescriptiva

\begin{tabular}{|c|c|c|c|c|c|}
\hline Variables predictoras & $\mathrm{B}$ & E. E. & $\beta$ & $t$ & $p$ \\
\hline Colectivismo & .132 & .024 & .250 & 5.430 & $<.001$ \\
\hline Normas & .358 & .076 & .208 & 4.703 & $<.001$ \\
\hline Ambiente familiar negativo & -.176 & .047 & -.158 & -3.746 & $<.001$ \\
\hline Ambiente familiar positivo & .035 & .016 & .099 & 2.198 & .028 \\
\hline$F$ & & & $(4,451)=31.446^{* * *}$ & & \\
\hline$R^{2} \mathrm{adj}$. & & & .212 & & \\
\hline
\end{tabular}

Nota. ${ }^{* * *} p<.001$.

Tabla 3.

Resumen del análisis de regresión lineal múltiple para las variables predictoras de la moral restrictiva

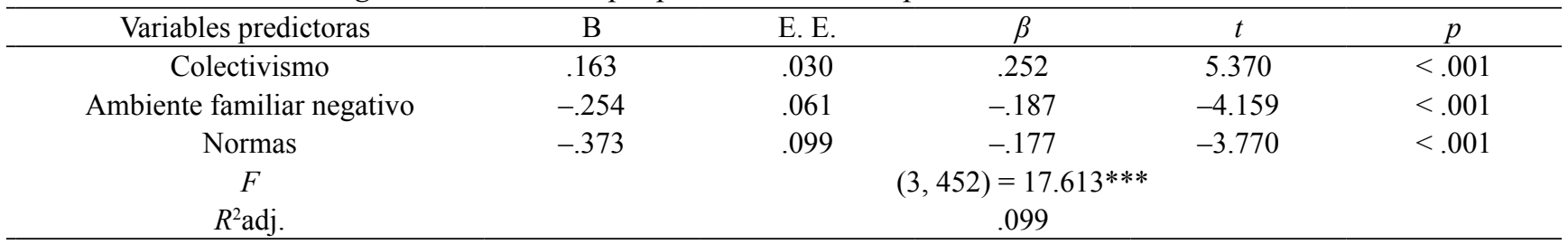

Nota. $* * * p<.001$. 
Figura 1.

Modelo sociocultural del dominio moral en adolescentes mexicanos

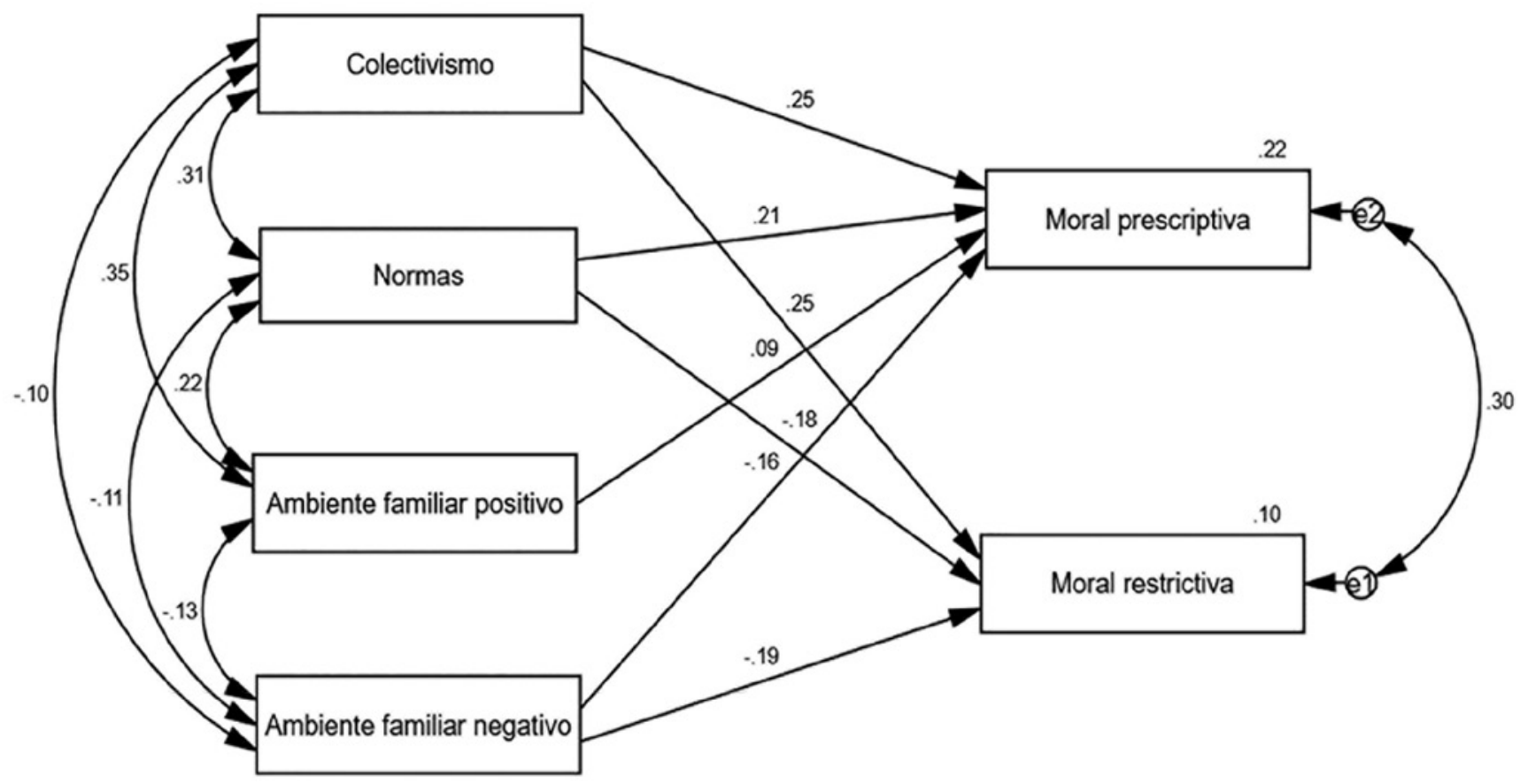

$\left(\chi^{2}=0.496, \mathrm{gl}=1, p=.481, \chi^{2} / \mathrm{gl}=0.496, \mathrm{AGFI}=.992\right.$, SRMR $=.006$, RMSEA $<.001)$.

Si bien las covarianzas entre errores no son recomendables debido a que muestran efectos no especificados por el modelo, también se puede asumir que estas covarianzas se deben a la presencia de otra variable que las converge (Ruiz et al., 2010). En el caso de la moral prescriptiva y la moral restrictiva, se asume que su covarianza entre errores se debe a que ambas pertenecen al constructo de dominio moral (Janoff-Bulman et al., 2009). Finalmente, tras estos análisis se obtiene un modelo de ecuaciones estructurales denominado modelo sociocultural del dominio moral en adolescentes mexicanos, que explica el $22 \%$ de la varianza de la moral prescriptiva y el $10 \%$ de la varianza de la moral restrictiva (véase Figura 1).

\section{Discusión}

Los objetivos de la investigación y las hipótesis se cumplieron a partir de los análisis de regresión lineal múltiple y la confirmación de un modelo estructural. Estos resultados permitieron reconocer que el carácter colectivista de la cultura, sus normas tradicionales, así como las características positivas y negativas del ambiente familiar, predicen el dominio moral prescriptivo y restrictivo en adolescentes mexicanos.
Como se pudo apreciar en los resultados, el colectivismo se muestra como la variable con mayor aporte en la explicación de las variaciones en el dominio moral en adolescentes. De este modo, así como los estudios de DíazGuerrero $(1994,2003)$ demuestran que el colectivismo marca las formas de comportarse de los mexicanos en lo sociocultural, los resultados de esta investigación demuestran que también aportan evidencia sobre lo moral, ya que el colectivismo es un predictor positivo de la moralidad de los adolescentes tanto en su componente prescriptivo como en el restrictivo, lo que quiere decir que, si se promueve en los adolescentes la consideración por los demás y la regulación del comportamiento en función de las expectativas sociales, probablemente aprenderán también la importancia tanto de orientarse hacia conductas de amor, respeto, lealtad y equidad, como de restringir conductas de ofensa y perjuicio.

A diferencia de los resultados en el estudio de Yilmaz et al. (2016), donde se pudo observar una distribución de individualismo y colectivismo con respecto a su relación con principios morales de una cultura diferente, en los adolescentes de la cultura mexicana evaluados en esta investigación predomina el colectivismo como promotor del dominio moral; algo que corresponde, a su vez, con las dinámicas de la cultura mexicana a través de sus premisas histórico-socio-culturales.

Por otra parte, los resultados demuestran lo que plantea Díaz-Loving (2011), en términos de que las PHSC marcan 
la orientación moral de las conductas. Particularmente, las PHSC, como normas, representan un referente que participa en la delimitación prescriptiva del dominio moral, aunque minimizan la delimitación restrictiva. De acuerdo con los resultados, las hipótesis también se confirman en el sentido de que las normas tradicionales de la cultura mexicana como predictoras positivas de la moral prescriptiva promueven el amor, el respeto, la lealtad a la familia y la equidad, pero como predictoras negativas de la moral restrictiva minimizan la importancia de restringir la ofensa y el perjuicio. Esto se relaciona con los planteamientos de Díaz-Guerrero (1994, 2003), en términos de que el mexicano prioriza el amor y la lealtad que se le tiene a la familia por encima incluso de la integridad individual. En este caso, se estima que los adolescentes mexicanos que más apoyen las normas tradicionales probablemente serán más propensos a ofender o perjudicar a personas ajenas a sus familias, ya sea por obediencia afiliativa, temor a la autoridad u honor familiar.

Lo anterior también se relaciona con los planteamientos de Bandura $(1999,2016)$ con respecto a la desconexión moral, donde se define que ciertas conductas perjudiciales $\mathrm{u}$ ofensivas se realizan sin remordimiento debido a que se justifican en supuestos criterios morales; en este caso, se aprecia una posible justificación de ofensas y perjuicios en normas culturales tradicionales por la prioridad que se le da a la familia. Por tanto, se sugiere que para la educación moral y sociocultural en adolescentes es fundamental promover el amor, la lealtad y la obediencia a través de normas culturales de la familia, pero también su cuestionamiento cuando tales normas conlleven a dañar a terceros, ya que para esta investigación se observa que la familia como eje de referencia para el comportamiento del mexicano se mantiene en los adolescentes encuestados.

Ahora bien, al considerar a la familia como el medio de la orientación cultural en los mexicanos, se identificó que el funcionamiento familiar también tiene un papel relevante en la distinción de lo bueno y lo malo para los adolescentes. Esto corresponde, a su vez, con hallazgos empíricos referidos por White y Matawie (2004), quienes indican que el ambiente familiar positivo tiene efectos positivos en el dominio moral de los adolescentes, por lo que se espera que, si perciben cohesión, comunicación y relaciones interpersonales armoniosas en su entorno familiar, probablemente también tendrán principios morales claros. Por otro lado, también se encontró efectos negativos de un ambiente familiar negativo en el dominio moral de los adolescentes encuestados, pues se encontró que, si perciben problemas interpersonales y ausencia de reglas en su entorno familiar, probablemente minimizarán la importancia de principios morales para realizar conductas de amor, respeto, lealtad y equidad, así como la necesidad de restringir conductas de ofensa o perjuicio. Como se puede observar, el funcionamiento familiar, aunado a las normas y valores sociales de la cultura mexicana colectivista, participan en el dominio moral de los adolescentes.

Finalmente, es importante resaltar que los valores estadísticos correlacionales y predictivos no son altos, y esto se puede deber al objetivo de elaborar un modelo con elementos únicamente sociales y culturales, pues se omitieron otros factores que también pueden participar en la predicción del dominio moral en adolescentes, como pueden ser las variables individuales propias de un desarrollo moral, como el razonamiento y la comprensión de situaciones en las que otras personas se encuentran involucradas, así como la edad y la escolaridad (Barba, 2001, 2002). Sin embargo, una de las bondades de un modelo sociocultural es que permite reconocer la variación de los principios morales de acuerdo con las costumbres y el entendimiento conceptual de las personas (Haste, 2013), además de que permite identificar la participación de aspectos particulares de una cultura en la conformación y en las diferencias con respecto a su dominio moral (Berniūnas et al., 2016).

Por tanto, se sugiere tomar en consideración aspectos individuales de los adolescentes para complementar la predicción de su dominio moral, sin olvidar la variabilidad cultural que aporta claridad sobre los principios que regulan su comportamiento en función de elementos que solo se pueden explicar por la cultura y el entorno particular en el que se desarrollan.

Otras aportaciones que brinda una perspectiva sociocultural del dominio moral se encuentran en reconocer principios morales que, si bien pueden ser compatibles con otras culturas, tienen conceptos con significados que solo son aplicables y relevantes para una sociedad, lo que se puede traducir en instrumentos de evaluación del desarrollo moral que sean sensibles a estos significados $y$, por ende, sean culturalmente relevantes. Esto puede dar claridad respecto a las diferencias por las que en instrumentos que parten de la teoría de Kohlberg (1987) los mexicanos tienen puntajes más bajos que los norteamericanos, y puede sugerir que se evalúe el desarrollo moral considerando principios que se relacionen con las normas y valores colectivistas de la cultura mexicana.

En conclusión, de acuerdo con los resultados de esta investigación, se sugiere que un modelo sociocultural del dominio moral en adolescentes aporta evidencia importante que, si se complementa con elementos de un desarrollo individual en los adolescentes, permitirá desarrollar una educación moral comprensible con sus pensamientos y sentimientos (Buxarrais-Estrada \& Martínez-Martín, 2009), de modo que se promueva un criterio moral consistente y un desarrollo saludable (Martin, 2006) para la etapa en la que se encuentran. 


\section{Referencias}

Bandura, A. (1999). Moral disengagement in the perpetration of inhumanities. Personality and Social Psychology Review, 3(3), 193-209. https://doi.org/10.1207/ s15327957pspr0303_3

Bandura, A. (2016). Moral disengagement: How people do harm and live with themselves. Worth Publishers.

Barba, B. (2001). Razonamiento moral de principios en estudiantes de secundaria y de bachillerato. Revista Mexicana de Investigación Educativa, 6(13), 501-523. https://www. comie.org.mx/revista/v2018/rmie/index.php/nrmie/article/ view/991/991

Barba, B. (2002). Influencia de la edad y de la escolaridad en el desarrollo del juicio moral. Revista Electrónica de Investigación Educativa, 4(2), 23-45. https://redie.uabc.mx/ index.php/redie/article/view/59/106

Barrios, A., Barbato, S., \& Branco, A. (2012). El análisis microgenético para el estudio del desarrollo moral: consideraciones teóricas y metodológicas. Revista de Psicología, 30(2), 249-279. https://doi.org/10.18800/psico.201202.002

Berniūnas, R., Dranseika, V., \& Sousa, P. (2016). Are there different moral domains? Evidence from Mongolia. Asian Journal of Social Psychology, 19(3), 275-282. https://doi. org/10.1111/ajsp.12133

Berry, W. D., \& Feldman, S. (1985). Multiple regression in practice. Sage Publications.

Buxarrais-Estrada, M. R., \& Martínez-Martín, M. (2009). Educación en valores y educación emocional: propuestas para la acción pedagógica. Teoría de la Educación. Educación y Cultura en la Sociedad de la Información, 10(2), 263-275. https://revistas.usal.es/index.php/eks/ article/view/7519/7552

Cohen, J., Cohen, P., West, S. G., \& Aiken, L. S. (2013). Applied multiple regression/correlation analysis for the behavioral sciences (3. ${ }^{\mathrm{a}}$ ed.). Erlbaum.

Díaz-Guerrero, R. (1972). Una escala factorial de premisas histórico-socioculturales de la familia mexicana. Revista Interamericana de Psicologia, (6), 235-244. https://journal. sipsych.org/index.php/IJP/article/view/646/548

Díaz-Guerrero, R. (1994). Psicología del mexicano (1. ${ }^{\mathrm{a}}$ ed.). Editorial Trillas.

Díaz-Guerrero, R. (2003). Bajo las garras de la cultura: psicología del mexicano 2 (1. ${ }^{\mathrm{a}}$ ed.). Editorial Trillas.

Díaz-Loving, R. (2011). Dinámica de las premisas históricosocio-culturales: Trayecto, vigencia y prospectiva. Revista Mexicana de Investigación en Psicología, 3(2), 174-180. https://www.revistamexicanadeinvestigacionenpsicologia.com/index.php/RMIP/article/view/143/56

Díaz-Loving, R., Rivera, S., Villanueva, G., \& Cruz, L. (2011). Las premisas histórico-socioculturales de la familia mexicana: su exploración desde las creencias y las normas. Revista Mexicana de Investigación en Psicología, 3(2), 128-142. https://www.revistamexicanadeinvestigacionenpsicologia. com/index.php/RMIP/article/view/137/50

García-Campos, T., Correa-Romero, F. E., García-Barragán, L. F., \& López-Suárez, A. D. (2016). Individualismocolectivismo y su efecto sobre la autoeficacia en jóvenes. Revista Mexicana de Psicología, 33(1), 71-79. https://www. redalyc.org/pdf/2430/243056043008.pdf

García-Méndez, M. (2007). Infidelidad y su relación con el poder y funcionamiento familiar: correlatos y predicciones (Tesis de doctorado no publicada). Universidad Nacional Autónoma de México.

García-Méndez, M., Rivera-Aragón. S., Reyes-Lagunes, I., \& Díaz-Loving, R. (2006). Construcción de una escala de funcionamiento familiar. Revista Iberoamericana de Diagnóstico y Evaluación Psicológica, 2(22), 91-110. https://www.redalyc.org/pdf/4596/459645449006.pdf

Graham, J., Haidt, J., Koleva, S., Motyl, M., Iyer, R., Wojcik, S., \& Ditto, P. (2013). Moral foundations theory: the pragmatic validity of moral pluralism. Advances in Experimental Social Psychology, 47(2013), 55-130. https:// doi.org/10.1016/B978-0-12-407236-7.00002-4

Haidt, J., \& Joseph, C. (2007). The moral mind: How 5 sets of innate moral intuitions guide the development of many culture-specific virtues, and perhaps even modules. En P. Carruthers, S. Laurence, and S. Stich (eds.) The Innate Mind, Vol. 3 (pp. 367-391). Oxford.

Haste, H. (2013). Deconstructing the elephant and the flag in the lavatory: Promises and problems of moral foundations research. Journal of Moral Education, 42(3), 316-329. https://doi.org/10.1080/03057240.2013.818529

Hofstede, G. (2001). Culture's consequences: Comparing values, behaviors, institutions and organizations across nations (2. ${ }^{\text {a }}$ ed.). Sage publications.

Hu, L. T., \& Bentler, P. M. (1999). Cutoff criteria for fit indexes in covariance structure analysis: Conventional criteria versus new alternatives. Structural Equation Modeling: A Multidisciplinary Journal, 6(1), 1-55. https:// doi.org/10.1080/10705519909540118

Jaen, C. (2017). Violencia durante el noviazgo y su impacto en la salud sexual y reproductiva de las y los adolescentes (Tesis de doctorado no publicada). Universidad Nacional Autónoma de México.

Janoff-Bulman, R., Sheikh, S., \& Hepp, S. (2009). Proscriptive Versus Prescriptive Morality: Two Faces of Moral Regulation. Journal of Personality \& Social Psychology, 96(3), 521-537. https://doi.org/10.1037/a0013779

Kohlberg, L. (1987). The development of moral judgment and moral action. En L. Kohlberg (Ed.). Child psychology and childhood education. A cognitive-developmental view (pp. 259-328). Longman.

Martin, M. W. (2006). From morality to mental health: Virtue and vice in a therapeutic culture (1. 'ed.). Oxford University Press. 
McKinney, J. P. (1971). The Development of Values. Prescriptive or Proscriptive? Human Development, 14(1), 71-80. https://doi.org/10.1159/000271055

Miles, J., \& Shevlin, M. (2001). Applying regression and correlation: A guide for students and researchers. Sage Publications.

Miranda-Rodríguez, R. A., \& García-Méndez, M. (2019). Construcción de una escala de dominio moral en adolescentes. Revista Iberoamericana de Diagnóstico y

Evaluación Psicológica, 53(4), 169-183. https://www.ai dep.org/sites/default/files/2019-10/RIDEP53-Art13.pdf

Railton, P. (1986). Moral realism. The Philosophical Review, 95(2), 163-207. http://www.jstor.com/stable/2185589

Rest, J. (1979). Developing in judging moral issues. University of Minnesota Press.

Ruiz, M. A., Pardo, A., \& San Martín, R. (2010). Modelos de ecuaciones estructurales. Papeles del psicólogo, 31(1), 3445. http://www.papelesdelpsicologo.es/pdf/1794.pdf

Shweder, R., Mahapatra, M., \& Miller, J. (1987). Culture and moral development. En J. Kagan y S. Lamb (Eds.), The emergence of morality in young children (pp. 1-83). University of Chicago Press.

Soler-Anguiano, L., \& Díaz-Loving, R. (2017). Validación de una Escala de Individualismo y Colectivismo. UARICHA Revista de Psicología, 14(32), 44-52. http://www.revis tauaricha.umich.mx/ojs_uaricha/index.php/urp/article/ view/150/135
Sullivan, D., Landau, M. J., Kay, A. C., \& Rothschild, Z. K. (2012). Collectivism and the meaning of suffering. Journal of Personality and Social Psychology, 103(6), 1023-1039. https://doi.org/10.1037/a0030382

Taibo, L. C., Gutiérrez, C. P., \& Muzzio, E. G. (2018). Graves vulneraciones de derechos en la infancia y adolescencia: Variables de funcionamiento familiar. Universitas Psychologica, 17(3), 1-13. https://doi.org/10.11144/ JAVERIANA.UPSY17-3.GVDI

Triandis, H. C. (1994). Culture and social behavior. Mc Graw Hill.

Turiel, E. (1983). The development of social knowledge: Morality and convention. Cambridge University Press.

White, F., \& Matawie, K. (2004). Parental morality and parental processes as predictors of adolescent morality. Journal of Child and Family Studies, 13(2), 219-233. https://doi. org/10.1023/B:JCFS.0000015709.81116.ce

Yilmaz, O., Harma, M., Bahcekapili, H., \& Cesur, S. (2016). Validation of the Moral Foundations Questionnaire in Turkey and its relation to cultural schemas of individualism and collectivism. Personality and Individual Differences, 99, 149-154. http://dx.doi.org/10.1016/j.paid.2016.04.090 